\title{
Correlative Microscopy: Elucidating the Mechanisms of SCC in Structural Alloys in PWR Environments
}

\author{
M. Grace Burke ${ }^{1}$, Liberato Volpe ${ }^{1}$, Fabio Scenini $^{1}$ and Eric Prestat $^{2}$ \\ ${ }^{1}$ University of Manchester, Manchester, Lancashire, England, United Kingdom, ${ }^{2}$ University of \\ Manchester and SuperSTEM Laboratory, Manchester, England, United Kingdom
}

The tremendous advances in electron microscopy and microanalysis have enabled significant advances in our understanding of environment-sensitive behaviour of materials used in nuclear power systems. Of particular importance has been the combined technique approach with a broad range of instruments from laser scanning confocal microscopes, FIB-SEM dual beam microscopes, low voltage SEMs, advanced analytical S/TEMs, etc. The new EDX spectrometer capabilities provided by low voltage Si Drift Detectors for use with low voltage SEM operation as well as by multiple SDD configurations for analytical electron microscopes have enabled nanoscale chemical analysis that approaches or rivals the atom probe. These advances coupled with the ability to study liquid-metal and gas-metal reactions are enabling fundamental materials research into the nanoscale reactions that are associated with degradation phenomena in power generation systems. These include the identifying the precursor reactions leading to the initiation of stress corrosion cracking in a susceptible annealed Ni-base alloy (Alloy 600), and the localized microstructural changes associated with material processing that can affect material susceptibility to SCC. Of particular interest is the related weld metal for Alloy 600, which is Alloy 182.

In these studies, FEG-SEM (Zeiss Merlin equipped with a STEM detector, an Oxford Instruments (OI) X-Max 150 and X-Max Extreme SDDs , and an EBSD detector with an Aztec analysis system), S/TEM (FEI Talos F200 with X-FEG and Super X (4 SDDs), and a probe-corrected 200 kV FEI Titan G2 S/TEM with X-FEG and Super X), FIB (FEI Helios 660 equipped with an OI X-Max 150 SDD) techniques were used to characterize the nature of the degradation phenomena. In addition, a Protochips Atmosphere system was used to examine whether the bulk observations could be reproduced in situ in the analytical electron microscope (Titan G2 S/TEM).

Alloy 600: Using an elevated temperature $\left(400-480^{\circ} \mathrm{C}\right) \mathrm{H}_{2}$-steam environment with a carefully controlled $\mathrm{O}_{2}$ partial pressure to generate reducing electrochemical conditions comparable those in the primary water environment of a Pressurized Water Reactor (PWR), it was possible to trace the nanoscale microstructural evolution leading to the preferential intergranular oxidation in solution-annealed and water-quenched Alloy 600. [1-3] An example, shown in Figure 1, includes a bright-field STEM image of partiallyoxidized grain boundary that has also experienced diffusion-induced grain boundary migration (DIGM), accompanied by pronounced asymmetric depletion of $\mathrm{Cr}$ and $\mathrm{Fe}$ behind the migrating grain boundary. The $\mathrm{Cr}$ and $\mathrm{Fe}$ diffused along the migrating boundary to form a $\mathrm{Cr}$-enriched and Fe-enriched oxides at the specimen surface and along the grain boundary. Detailed TEM characterization revealed the presence of very fine, discrete $\mathrm{Cr}$-rich $\mathrm{M}_{23} \mathrm{C}_{6}$ carbides that marked the location of the original grain boundary prior to specimen exposure in the test environment. Additional STEM-EDX analyses revealed the presence of $\mathrm{Al}$ and $\mathrm{Ti}$ associated with the migrated grain boundaries and the preferential intergranular oxides.

Using "thick" (250-300 nm) "hybrid" [4] specimens with the Protochips Atmosphere in situ system, it was possible to generate comparable DIGM to that observed in bulk ex situ samples [5]. Finally, by exposing stressed $4 \mathrm{pt}$. bend samples in the same $480^{\circ} \mathrm{C} \mathrm{H}_{2}$-steam reducing environment, it was possible 
to generate intergranular fracture along the preferentially oxidized grain boundaries, thereby linking the nanoscale microstructural development with the nucleation of a small stress corrosion crack. [6]

Alloy 182 Welds: As part of the ongoing collaborative European Horizon 2020 program MEACTOS (Mitigation of EAC Through the Optimization of Surfaces), detailed microstructural characterization coupled with an extensive SCC test program is exploring the effect of various surface treatments on SCC susceptibility to identify potential SCC mitigation strategies that can be employed to optimize performance of these materials. Of particular interest are various machining operations, including advanced machining methods, that can minimize the extent of surface/near-surface deformation in these materials. Thus, microstructural analyses, including Transmission Kikuchi Diffraction (TKD), TEM and EBSD characterization are of great importance.

Research to date had demonstrated that the advanced surface machining process induced an ultrafinegrained layer adjacent to the as-machined surface, which extended approximately 2 microns into the weld. These ultrafine grains ranged in size from $20 \mathrm{~nm}$ to $\sim 200 \mathrm{~nm}$, as shown in the TKD IPF of Figure 2 (a-b). Beneath the ultrafine-grained layer was another deformed zone with more pancaked deformed grains that extended a further 2 to 3 microns into the weld. The TEM montage in Figure 2c illustrates the pancaked grain structure approximately 3 to 5 microns from the as-machined surface. Further results on the effect of different surface treatments for SCC mitigation will be discussed, for both Ni-base alloys and austenitic stainless steel. [7]

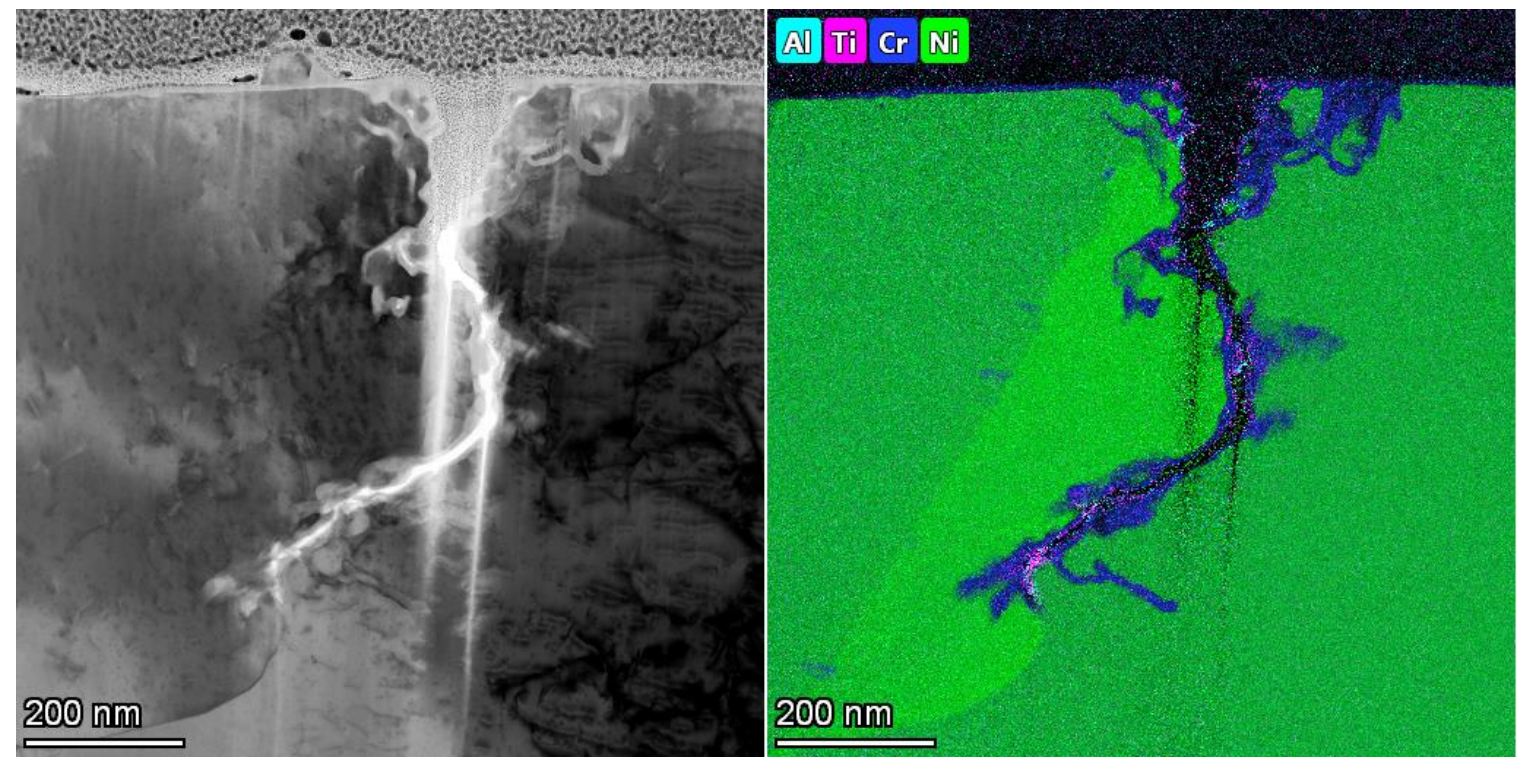

Figure 1. BF STEM image and the combined STEM-EDX elemental map for Cr, Al, Ti and Ni, illustrating the pronounced DIGM with solute asymmetry and the presence of the $\mathrm{Cr}$-rich intergranular oxide containing local enrichments of $\mathrm{Al}$ and $\mathrm{Ti}$. Note the presence of fine $\mathrm{Cr}$-rich precipitates, which were identified by selected area electron diffraction as M23C6, that formed along the original grain boundary. 


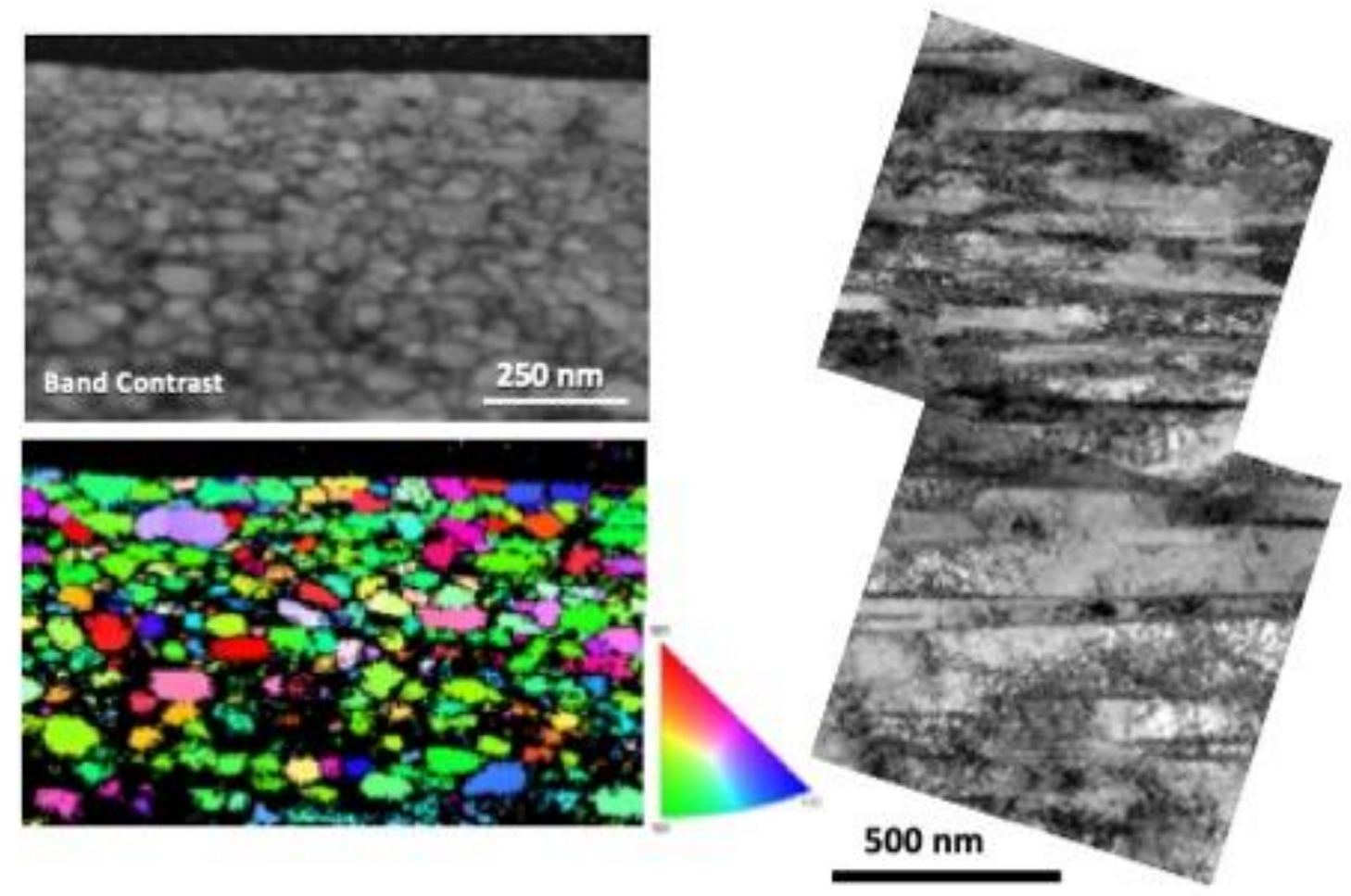

Figure 2. (a) TKD Band Contrast map and (b) inverse pole figure showing the variation in grain size within the ultrafine-grained near-surface layer in advanced machined Alloy 182 weld metal. (c) BF TEM montage of the pancaked deformed grains beneath the ultrafine-grained layer.

\section{References}

[1] G. Bertali et al., Corr. Sci., 100 (2015) 474-483.

[2] G. Bertali et al., Met. Mat. Trans. A, 49 (2018) 1879-1894.

[3] L. Volpe et al., Acta Mat. 186 (2020) 454-466.

[4] X.L. Zhong et al., Microsc. Microanal. 22 (2016) 1350-7

[5] M.G. Burke et al., Ultramicroscopy, 176 (2017) 46-51.

[6] L. Volpe et al., Acta Mat. 175 (2019) 238-249.

[7] MGB acknowledges support from EPSRC EP/N017854/1. The MEACTOS project receives funding from the Euratom research and training programme 2014-2018 under grant agreement no. 755151. The views and opinions expressed herein do not necessarily reflect those of the European Commission. 